\title{
A study on fractional differential equations using the fractional Fourier transform
}

\author{
Porpattama Hammachukiattikul', Arusamy Mohanapriya², Anumanthappa Ganesh³, \\ Grienggrai Rajchakit ${ }^{4 *}$ (D) Vediyappan Govindan ${ }^{5}$, Nallappan Gunasekaran ${ }^{6}$ and Chee Peng Lim
}

"Correspondence:

kreangkri@mju.ac.th

${ }^{4}$ Department of Mathematics,

Faculty of Science, Maejo University,

Sansai, 50290 Chiang Mai, Thailand

Full list of author information is

available at the end of the article

\section{Springer}

\begin{abstract}
This study aims to use the fractional Fourier transform for analyzing various types of Hyers-Ulam stability pertaining to the linear fractional order differential equation with Atangana and Baleanu fractional derivative. Specifically, we establish the Hyers-Ulam-Rassias stability results and examine their existence and uniqueness for solving nonlinear problems. Simulation examples are presented to validate the results.

Keywords: Hyers-Ulam-Rassias stability; Fourier transform; Mittag-Leffler kernel; Caputo-Fabrizio fractional differential equation
\end{abstract}

\section{Introduction}

A fractional order differential equation (FODE) is a generalized form of an integer order differential equation. The FODE is useful in many areas, e.g., for the depiction of a physical model of various phenomena in pure and applied science (see [1-4] and the references therein). The resulting equations offer inconceivable thought for researchers and analysts.

The first definition of the fractional derivative was introduced at the end of nineteenth century by Liouville and Riemann, but the concept of noninteger derivative and integral was mentioned already in 1695 by Leibniz and L'Hospital. Actually, FODEs are considered as an alternative model to integer differential equation. The definition of RiemannLiouville derivative was established by Riemann in 1876 . Since then many applications of the fractional derivatives and integrals of this Riemann-Liouville type have been demonstrated in numerous fields of science and technology. These include studies on controllability, stokes problem, thermoelasticity, vibration, and diffusion processes, bioengineering issues, and other complex phenomena (see [5-7]).

A current research topic is focused on understanding the various properties of fractional derivatives and their effectiveness in certain complex systems. This research direction hinges on the results reported by numerous researchers on complex systems of nonlocal elements, i.e., a local operator of ordinary derivative leads to a nonlocal fractional operator. Along these research lines, it is worthwhile to study fractional-order models in terms of global optimization. Toward the start of the twentieth century, another definition of fractional derivative was proposed by Caputo in regard to a Riemann-Liouville fractional

(c) The Author(s) 2020. This article is licensed under a Creative Commons Attribution 4.0 International License, which permits use sharing, adaptation, distribution and reproduction in any medium or format, as long as you give appropriate credit to the original author(s) and the source, provide a link to the Creative Commons licence, and indicate if changes were made. The images or other third party material in this article are included in the article's Creative Commons licence, unless indicated otherwise in a credit line to the material. If material is not included in the article's Creative Commons licence and your intended use is not permitted by statutory regulation or exceeds the permitted use, you will need to obtain permission directly from the copyright holder. To view a copy of this licence, visit http://creativecommons.org/licenses/by/4.0/. 
integral. Caputo and Fabrizio [8] presented another definition of nonlocal derivative without singular kernel, i.e.,

$$
{ }^{\mathcal{C F}} \mathcal{D}_{a}^{v} \mathcal{U}(t)=\frac{\mathcal{B}(v)}{1-v} \frac{d}{d t} \int_{a}^{t} \exp \left(\frac{-v}{1-v}(t-s)^{v}\right) \mathcal{U}(s) d s,
$$

which is valid for $0<v<1$, with a normalization function of $\mathcal{B}(v)$ that satisfies $\mathcal{B}(0)=$ $\mathcal{B}(1)=1$.

Another operator of fractional order with respect to the generalized Mittag-Leffler function has been proposed [9]. The key point is to evaluate if another kind of fractional operator having nonsingular kernel can better represent the dynamics of nonlocal phenomena. We generally assume created definitions of fractional integrals address a similar fundamental test per the study in [8]. Specifically, the Mittag-Leffler function, which is a nonlocal and nonsingular kernel, is exploited, i.e.,

$$
{ }^{\mathcal{A B R}} \mathcal{D}_{a}^{v} \mathcal{U}(t)=\frac{\mathcal{B}(v)}{1-v} \frac{d}{d t} \int_{a}^{t} \mathcal{U}(s) E_{v}\left(\frac{-v}{1-v}(t-s)^{v}\right) d s
$$

and

$$
{ }^{\mathcal{A B C}} \mathcal{D}_{a}^{v} \mathcal{U}(t)=\frac{\mathcal{B}(v)}{1-v} \int_{a}^{t} \mathcal{U}^{\prime}(s) E_{\nu}\left(\frac{-v}{1-v}(t-s)^{v}\right) d s
$$

for $0<v<1$, where $\mathcal{B}(v)$ is a normalization function, $\mathcal{A B R}$ and $\mathcal{A B C}$ are the Atangana and Baleanu fractional derivatives with respect to the Riemann-Liouville and Caputo sense. Along these lines, we can examine various dynamics of nonlocal complex systems. The new Atangana and Baleanu $\mathcal{A B}$ formulation has been investigated in different mathematical models, e.g., in heat transfer, chaos theory, and variational problems [10-13].

It is now well accepted that the Mittag-Leffler function is exceptionally helpful in the area of fractional calculus, serving as an effective method for analytical expression pertaining to the solution of differential equations with integer or noninteger order. Swedish mathematician G.M. Mittag-Leffler introduced the Mittag-Leffler function in 1902 [14] with respect to the generalization of exponential function, and it plays an important role in fractional calculus. The generalization and properties have been considered and discussed in [15-17]. From the literature, the complementary role between the Mittag-Leffler nonsingular kernel and classical fractional calculus is well established, offering the capability of analyzing various properties in nonlocal dynamical systems. In many practical problems, it is a great challenge to formulate an exact solution for certain differential equation of a physical model. Powerful numerical or analytical principles with algorithms and methods that can produce stable outcomes are necessary. In this case, stability analysis is used, which forms an incredibly obvious part of differential equation. It is important to discuss the approximate solution and determine whether it lies near the exact solution. In general, we confirm the stability of a differential equation if, for each solution of a troubled equation, an approximate solution nearby the exact solution exists. In the literature, there exist different types of stability, but recently the concept of Hyers-Ulam stability is a central topic for researchers because it is very important in approximation theory. The historical background of the Hyers-Ulam stability stems from the nineteenth century. Ulam [18] detailed a class of stability with respect to a functional equation, which was tackled by Hyers 
[19] for an additive function defined on the Banach space. After this result, Rassias generalized the stability idea [20], which resulted in the Hyers-Ulam-Rassias stability. Furthermore, Obloza [21] studied the Hyers-Ulam stability with respect to linear differential equation for the first time, and Alsina and Ger [22] later investigated the Hyers-Ulam stability of differential equation. There are many advantages of Hyers-Ulam type stability in tackling problems, related to optimization techniques, numerical analysis, control theory, and many more. Further advances in the Hyers-Ulam stability of differential equation can be found in [23-33].

The idea of Fourier transform was first suggested by French mathematician Jean Baptiste Joseph Fourier in 1807. The fractional Fourier transform is a generalization of the ordinary Fourier transform. Every property and application of the common Fourier transform becomes a special case of the fractional Fourier transform. The fractional Fourier transform was introduced by Wiener [34] as a way to solve certain types of ordinary and partial differential equations arising in quantum mechanics. Unaware of Wiener's work, Victor Namias [35] proposed the fractional Fourier transform also to solve differential equations in quantum mechanics from classical quadratic Hamiltonian. His results were later refined by McBride, and Kerr [36] developed an operational calculus for the transform. The fractional Fourier transform can be used to solve ordinary and partial differential equations as well as fractional and integral equations.

The flow chart of fractional Fourier transform is represented in Fig. 1.

It is well known that the analysis of stability of FODE is more complex than that of classical differential equations, since fractional derivatives are nonlocal and have weak kernels. As a result, the development of stability of nonlinear FODE is a bit slow. Recently, Liu et al. [37] discussed the stability of the following FODE:

$$
\left({ }^{\mathcal{A B C}} \mathcal{D}_{+}^{v} \mathcal{U}(t)\right)-\lambda\left({ }^{\mathcal{A B C}} \mathcal{D}_{+}^{\alpha} \mathcal{U}(t)\right)=\mathcal{G}(t), \quad t \in[0, b]
$$

where $0<v, \alpha<1$, and $\lambda \in \mathbb{R}$. In addition, the study on existence and uniqueness for solution to the following nonlinear FODE was conducted:

$$
\left({ }^{\mathcal{A B C}} \mathcal{D}_{+}^{\nu} \mathcal{U}(t)\right)-\lambda\left({ }^{\mathcal{A B C}} \mathcal{D}_{+}^{\alpha} \mathcal{U}(t)\right)=\mathcal{G}(t, \mathcal{U}(t)), \quad t \in[0, b],
$$

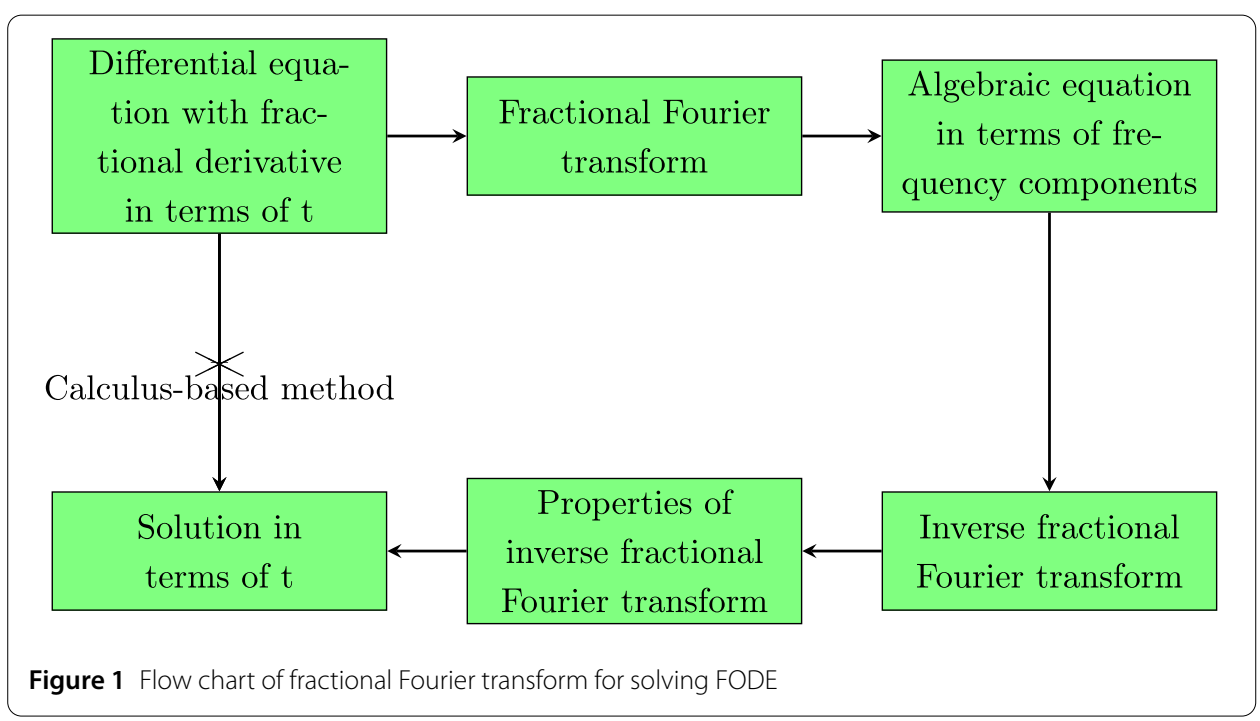


and the stability result was obtained by using the Laplace transform method. In [38], the stability of FODE with respect to the generalized Caputo-Fabrizio fractional derivative was discussed. Although, there is some work on the stability of FODE with Mittag-Leffler kernel, to the best of our knowledge, there are no outcomes on the Ulam stability of FODEs by using fractional Fourier transform, which may provide a new way for the researchers to investigate the stability of FODEs from different perspective.

In this paper, we examine the existence of the Hyers-Ulam stability as well as HyersUlam-Rassias stability of linear FODE

$$
{ }^{\mathcal{A B R}} \mathcal{D}_{\beta}^{\nu} \mathcal{U}(t)=\mathcal{G}(t), \quad \forall t \in \mathbb{R},
$$

where $\mathcal{U}(t)$ is a continuously differential function. We develop the relevant results that satisfy the condition of Hyers-Ulam stability by using fractional Fourier transform, which provides a new method to study such problems. Specifically, we analyze the Hyers-UlamRassias stability of the nonlinear FODE

$$
{ }^{\mathcal{A B R}} \mathcal{D}_{\beta}^{v} \mathcal{U}(t)=\mathcal{G}(t, \mathcal{U}(t)), \quad \forall t \in \mathbb{R}
$$

and use the fixed point theorems of Banach and Schaefer for examining the existence and uniqueness of the solutions.

In Sect. 2, some definitions, lemmas, and hypotheses are presented. The Hyers-Ulam stability of linear FODE is introduced in Sect. 3. In Sect. 4, the existence, uniqueness, and stability of the solutions pertaining to nonlinear FODE are presented. Numerical examples and conclusions are included in Sects. 5 and 6, respectively.

\section{Preliminaries}

In this paper, $\mathbb{F}$ represents real field $\mathbb{R}$ or complex field $\mathbb{F}$. For a function $\mathcal{U} \in \mathcal{S}, \mathcal{S}$ is the space of rapidly decreasing functions on the real axis $\mathbb{R}$. The Fourier transform and inverse Fourier transform of function $\mathcal{U}(t)$ are defined as follows:

$$
\begin{aligned}
& \widehat{\mathcal{U}}(\vartheta)=(\mathcal{F} \mathcal{U}(t))(\vartheta)=\int_{-\infty}^{\infty} \mathcal{U}(t) e^{i \vartheta t} d t, \quad \vartheta \in \mathbb{R}, \\
& \mathcal{U}(t)=\left(\mathcal{F}^{-1} \widehat{\mathcal{U}}(\vartheta)\right)(t)=\frac{1}{2 \pi} \int_{-\infty}^{\infty} \widehat{\mathcal{U}}(\vartheta) e^{-i \vartheta t} d \vartheta, \quad t \in \mathbb{R},
\end{aligned}
$$

where $(t, \vartheta)$ are space and frequency variable, respectively, and the integration paths run along the real axes $t \in(-\infty, \infty)$ and $\vartheta \in(-\infty, \infty)$. The operator $\widehat{\mathcal{U}}$ can be defined on the spaces $L_{p}(\mathbb{R}), 1 \leq p \leq 2$. The Fourier transform of the function $\mathcal{U}(t)$ is defined only when improper integral converges. It can be used to solve differential equations, ordinary and partial, as well as fractional and integral equations.

For working with fractional Fourier transform and with fractional differentiation and integration operators, some other spaces of functions are usually employed. In this paper, we say in the framework of the Lizorkin space of function (see [39-41]). Its definition is provided below.

Definition 2.1 Let us denote by $V(\mathbb{R})$ the set of functions $v \in \mathcal{S}$ satisfying the conditions

$$
\left.\frac{d^{n} v}{d t^{n}}\right|_{t=0}=0, \quad n=0,1,2,3, \ldots
$$


The Lizorkin space $\Phi(\mathbb{R}) \subset L^{1}(\mathbb{R})$ is defined as the Fourier pre-image of the space $V(\mathbb{R})$ in the space $\mathcal{S}$, i.e.,

$$
\Phi(\mathbb{R})=\{\mathcal{U} \in \mathcal{S} ; \mathcal{F}(\mathcal{U}) \in V(\mathbb{R})\} .
$$

According to the definition of the Lizorkin space, the orthogonality condition is satisfied by any function $\mathcal{U}(t) \in \Phi(\mathbb{R})$, i.e.,

$$
\int_{-\infty}^{\infty} t^{n} \mathcal{U}(t) d t=0, \quad n=0,1,2,3, \ldots
$$

It is known that the Lizorkin space $\Phi(\mathbb{R})$ is invariant with respect to the fractional integration and differentiation operator (this is not the case for the whole space $\mathcal{S}$ of the rapidly decreasing functions because the fractional integrals and derivatives of the functions from the space $\mathcal{S}$ do not always belong to the space $\mathcal{S}$ ).

Definition 2.2 ([42]) The definition of a fractional Fourier transform (FRFT) of a function $\mathcal{U} \in \Phi(\mathbb{R})$ of order $v,(0<v \leq 1)$ is

$$
\widehat{\mathcal{U}}_{v}(\vartheta)=\left(\mathcal{F}_{\nu} \mathcal{U}(t)\right)(\vartheta)=\int_{-\infty}^{\infty} \mathcal{U}(t) e_{v}(\vartheta, t) d t
$$

where

$$
\begin{aligned}
& e_{v}(\vartheta, t)=\left\{\begin{array}{ll}
e^{-i|\vartheta|^{1 / v}} t ; & \vartheta \leq 0, \\
e^{i|\vartheta|^{1 / v}} t ; & \vartheta \geq 0
\end{array}=e^{i \operatorname{sign}(\vartheta)|\vartheta|^{1 / v} t},\right. \\
& \operatorname{sign}(\vartheta)= \begin{cases}-1 ; & \vartheta<0, \\
1 ; & \vartheta \geq 0 .\end{cases}
\end{aligned}
$$

If $v=1$, the FRFT is concurring with Fourier transform. Function $\mathcal{U} \in \Phi(\mathbb{R})$ has an inverse fractional Fourier transform in the form of

$$
\mathcal{U}(t)=\frac{1}{2 \pi v} \int_{-\infty}^{\infty} e^{-i \operatorname{sign}(\vartheta)|\vartheta|^{1 / v} t}|\vartheta|^{\frac{1}{v}-1} \widehat{\mathcal{U}}_{v}(\vartheta) d \vartheta,
$$

for any $t \in \mathbb{R}$ and $v>0$, the relation

$$
\mathcal{F}^{-1} \mathcal{F}(\mathcal{U})=\mathcal{U}
$$

holds true almost everywhere on $\mathbb{R}$.

Definition 2.3 The function $\left(\mathcal{U}_{1} * \mathcal{U}_{2}\right)(t)=\int_{\mathbb{R}} \mathcal{U}_{1}(t-\tau) \mathcal{U}_{2}(\tau) d \tau$ is called the convolution of both functions $\mathcal{U}_{1}$ and $\mathcal{U}_{2}$ defined on $\mathbb{R}$.

Some properties of FRFT that are closely related to the solution are:

1 If $\left(\mathcal{F}_{\nu} \mathcal{U}_{1}(t)\right)(\vartheta)=\left(\mathcal{F}_{\nu} \mathcal{U}_{2}(t)\right)(\vartheta)$, then $\mathcal{U}_{1}(t)=\mathcal{U}_{2}(t), \forall t \in \mathbb{R}$;

$2 F\left(\mathcal{F}_{v} \mathcal{U}(t-\xi)\right)(\vartheta)=e_{\nu}(\vartheta, \xi) \widehat{\mathcal{U}}(\vartheta)$;

$3 \mathcal{F}_{v}\left(\mathcal{U}_{1}(t) * \mathcal{U}_{2}(t)\right)(\vartheta)=\mathcal{F}_{v}\left(\mathcal{U}_{1}(t)\right)(\vartheta) \mathcal{F}_{v}\left(\mathcal{U}_{2}(t)\right)(\vartheta) ;$

4. $\mathcal{F}_{v}^{-1}\left(\mathcal{U}_{1} \mathcal{U}_{2}\right)(t)=\mathcal{F}_{v}^{-1}\left(\mathcal{U}_{1}\right)(t) * \mathcal{F}_{v}^{-1}\left(\mathcal{U}_{2}\right)(t)$. 
Definition 2.4 ([43]) The definition of the Mittag-Leffler function is

$$
E_{v}(t)=\sum_{m=0}^{\infty} \frac{t^{m}}{\Gamma(v m+1)}, \quad 0<v \leq 1
$$

The above function with one parameter can be extended to a general function consisting of two parameters, i.e.,

$$
E_{v, \beta}(t)=\sum_{m=0}^{\infty} \frac{t^{m}}{\Gamma(v m+\beta)}, \quad 0<v, \beta \leq 1 .
$$

Definition 2.5 ([44]) The definition of the left- and right-hand side Riemann-Liouville fractional integral with order $v>0$ pertaining to function $\mathcal{U}(t)$ is

$$
\left({ }^{\mathrm{RL}} \mathcal{I}_{+}^{\nu} \mathcal{U}\right)(t)=\frac{1}{\Gamma \nu} \int_{-\infty}^{t}(t-s)^{\nu-1} \mathcal{U}(s) d s \quad(\text { Left RLI })
$$

and

$$
\left({ }^{\mathrm{RL}} \mathcal{I}_{-}^{\nu} \mathcal{U}\right)(t)=\frac{1}{\Gamma \nu} \int_{t}^{\infty}(s-t)^{\nu-1} \mathcal{U}(s) d s \quad(\text { Right RLI })
$$

where $\operatorname{Re}(v)>0$, we have $\Gamma v=\int_{0}^{\infty} e^{-t} t^{\nu-1} d t$.

Definition 2.6 ([44]) Given function $\mathcal{U} \in \Phi(\mathbb{R})$, the definition of the Riemann-Liouville derivative with fractional order $v>0, m=[v]+1$ is

$$
\mathcal{D}_{+}^{v} \mathcal{U}(t)=\left(\frac{d}{d t}\right)^{m} \mathcal{I}_{+}^{m-v} \mathcal{U}(t) \quad(\text { Left } \mathrm{RLD})
$$

and

$$
\mathcal{D}_{-}^{v} \mathcal{U}(t)=\left(-\frac{d}{d t}\right)^{m} \mathcal{I}_{-}^{m-v} \mathcal{U}(t) \quad \text { (Right RLD), }
$$

provided that it exists. Here, the integer part of real number $v$ is denoted by $[v]$.

Definition 2.7 ([9]) Let $0<v<1$, and a function $\mathcal{U} \in \Phi(\mathbb{R})$. The definition of the left- and right-hand side of $\mathcal{A B R}$ fractional derivative is

$$
{ }^{\mathcal{A B R}} \mathcal{D}_{+}^{v} \mathcal{U}(t)=\frac{\mathcal{B}(v)}{1-v} \frac{d}{d t} \int_{-\infty}^{t} \mathcal{U}(s) E_{v}\left(\frac{-v}{1-v}(t-s)^{v}\right) d s \quad \text { (Left ABRD) }
$$

and

$$
{ }^{\mathcal{A B R}} \mathcal{D}_{-}^{v} \mathcal{U}(t)=\frac{\mathcal{B}(v)}{1-v} \frac{d}{d t} \int_{t}^{\infty} \mathcal{U}(s) E_{v}\left(\frac{-v}{1-v}(s-t)^{v}\right) d s \quad \text { (Right ABRD) }
$$

where $\mathcal{B}(v)$ is a normalization function. More details on the $\mathcal{A B R}$ fractional derivative can be found in $[45,46]$. 
Definition 2.8 ([9]) Given $0<v<1$ and function $\mathcal{U} \in \Phi(\mathbb{R})$, the definition of the left and right $\mathcal{A B}$ fractional integral is

$$
{ }^{\mathcal{A}} \mathcal{I}_{+}^{v} \mathcal{U}(t)=\frac{1-v}{\mathcal{B}(v)} \mathcal{U}(t)+\frac{v}{\mathcal{B}(v) \Gamma(v)} \int_{-\infty}^{t} \mathcal{U}(t)(t-s)^{\nu-1} d s \quad \text { (Left ABI) }
$$

and

$$
{ }^{\mathcal{A}} \mathcal{I}_{-}^{v} \mathcal{U}(t)=\frac{1-v}{\mathcal{B}(v)} \mathcal{U}(t)+\frac{v}{\mathcal{B}(v) \Gamma(v)} \int_{t}^{\infty} \mathcal{U}(t)(s-t)^{v-1} d s \quad \text { (Right ABI) }
$$

Lemma 2.1 (Integration by parts; [47]) For any functions $\mathcal{U}(t), \mathcal{V}(t) \in \Phi(\mathbb{R})$, we have

$$
\begin{aligned}
& \int_{-\infty}^{\infty} \mathcal{U}(t)^{\mathcal{A B R}} \mathcal{D}_{+}^{\nu} \mathcal{V}(t) d t=\int_{-\infty}^{\infty} \mathcal{V}(t)^{\mathcal{A B R}} \mathcal{D}_{-}^{\nu} \mathcal{U}(t) d t \\
& \int_{-\infty}^{\infty} \mathcal{U}(t)^{\mathcal{A B R}} \mathcal{D}_{-}^{\nu} \mathcal{V}(t) d t=\int_{-\infty}^{\infty} \mathcal{V}(t)^{\mathcal{A B R}} \mathcal{D}_{+}^{\nu} \mathcal{U}(t) d t
\end{aligned}
$$

Theorem 2.1 (Arzela-Ascoli's theorem; [48])

(1) A family $\mathcal{B}$ of continuous functions on $\mathbb{I}=[a, b]$ is a uniformly bounded set if there exists $\lambda>0$ with

$$
\|\mathcal{U}\|=\sup |\mathcal{U}(t)|<\lambda, \quad \forall \mathcal{U} \in \mathcal{B} .
$$

(2) $\mathcal{B}$ is an equicontinuous set, i.e., for any $\epsilon>0$, there exists $\delta>0$ such that

$$
\left|t_{1}-t_{2}\right| \leq \delta \Rightarrow\left|\mathcal{U}\left(t_{1}\right)-\mathcal{U}\left(t_{2}\right)\right| \leq \epsilon, \quad \forall \mathcal{U} \in \mathcal{B}
$$

Let $\left\{u_{n}\right\}_{n \in N}$ be a family of continuous functions on $\mathbb{I}=[a, b]$. If the sequence is uniformly bounded and equicontinuous, then there exists a subsequence $\left\{u_{n_{1}}(t)\right\}_{n_{1} \in N}$ that converges uniformly.

Theorem 2.2 (Banach fixed point theorem; [49]) Let $\mathcal{B}$ be a nonempty closed subset of a Banach space $\psi$. Then any contraction mapping $\Delta$ from $\psi$ into itself has a unique fixed point.

Theorem 2.3 (Schaefer's fixed point theorem; [49]) A Banach space is denoted as $\psi$, and a completely continuous mapping is $\Delta: \psi \rightarrow \psi$. As such, if

$$
\mathcal{B}=\{\mathcal{U} \in \psi \mid \mathcal{U}=\theta \Delta \mathcal{U}, 0<\theta<1\}
$$

is a bounded set, then there is at least one fixed point on $\psi$ in $\Delta$.

Lemma 2.2 ([50]) Suppose that $\mathcal{U} \in \Phi(\mathbb{R})$ is a continuously differential function, $0<v \leq 1$, and $\vartheta \in \mathbb{R} /\{0\}$, then

$$
{ }^{\mathrm{RL}} \mathcal{I}_{+}^{\nu m+1}\left(e^{i \operatorname{sign}(\vartheta)|\vartheta|^{\frac{1}{v}} t}\right)=e^{i \operatorname{sign}(\vartheta)|\vartheta|^{\frac{1}{v} t}}|\vartheta|^{\frac{-(v m+1)}{\nu}} e^{-i \operatorname{sign}(\vartheta)(1+v m) \pi / 2} .
$$


Proof By taking into account the Riemann-Liouville fractional integral equation (2.1), we get

$$
{ }^{\mathrm{RL}} \mathcal{I}_{+}^{v m+1}\left(e^{i \operatorname{sign}(\vartheta)|\vartheta|^{\frac{1}{v}} t}\right)=\frac{1}{\Gamma(1+v m)} \int_{-\infty}^{t}(t-s)^{1+\nu m-1} e^{i \operatorname{sign}(\vartheta)|\vartheta|^{\frac{1}{v}}} s d s,
$$

using the variables substitution $\eta=t-s$, we obtain

$$
\begin{aligned}
&{ }^{\mathrm{RL}} \mathcal{I}_{+}^{v m+1}\left(e^{i \operatorname{sign}(\vartheta)|\vartheta|^{\frac{1}{v}} t}\right) \\
&=\frac{1}{\Gamma(1+\nu m)} e^{i \operatorname{sign}(\vartheta)|\vartheta|^{\frac{1}{v}} t} \int_{0}^{\infty} \eta^{\nu m} e^{-i \operatorname{sign}(\vartheta)|\vartheta|^{\frac{1}{v}} \eta} d \eta \\
&=e^{i \operatorname{sign}(\vartheta)|\vartheta|^{\frac{1}{v}} t} \frac{1}{\Gamma(1+\nu m)} \int_{0}^{\infty} \eta^{\nu m} \cos \left(|\vartheta|^{\frac{1}{v}} \eta\right) d \eta \\
&-i \operatorname{sign}(\vartheta) e^{i \operatorname{sign}(\vartheta)|\vartheta|^{\frac{1}{v}} t} \frac{1}{\Gamma(1+\nu m)} \int_{0}^{\infty} \eta^{\nu m} \sin \left(|\vartheta|^{\frac{1}{v}} \eta\right) d \eta
\end{aligned}
$$

Both the integrals in the above equation can be evaluated from using the integral formula in [51]:

$$
\begin{aligned}
& \frac{1}{\Gamma(1+\nu m)} \int_{0}^{\infty} \eta^{(\nu m+1)-1} \cos \left(|\vartheta|^{\frac{1}{v}} \eta\right) d \eta=|\vartheta|^{\frac{-(1+v m)}{v}} \cos ((1+v m) \pi / 2), \\
& \frac{1}{\Gamma(1+\nu m)} \int_{0}^{\infty} \eta^{(\nu m+1)-1} \sin \left(|\vartheta|^{\frac{1}{v}} \eta\right) d \eta=\operatorname{sign}(\vartheta)|\vartheta|^{\frac{-(1+v m)}{v}} \sin ((1+\nu m) \pi / 2) .
\end{aligned}
$$

Substituting equation (2.4) and (2.5) in equation (2.3), we get

$$
{ }^{\mathrm{RL}} \mathcal{I}_{+}^{\nu m+1}\left(e^{i \operatorname{sign}(\vartheta)|\vartheta|^{\frac{1}{v}} t}\right)=e^{i \operatorname{sign}(\vartheta)|\vartheta|^{\frac{1}{v} t}}|\vartheta|^{\frac{-(v m+1)}{\nu}} e^{-i \operatorname{sign}(\vartheta)(1+\nu m) \pi / 2} .
$$

Proceeding in a similar way, we prove the next lemma.

Lemma 2.3 ([50]) Suppose that $\mathcal{U} \in \Phi(\mathbb{R})$ is a continuously differential function, $0<v \leq 1$, and $\vartheta \in \mathbb{R} /\{0\}$, then

$$
\mathrm{RL}_{\mathcal{I}_{-}^{v m+1}}\left(e^{i \operatorname{sign}(\vartheta)|\vartheta|^{\frac{1}{v}} t}\right)=e^{i \operatorname{sign}(\vartheta)|\vartheta|^{\frac{1}{v} t}}|\vartheta|^{\frac{-(v m+1)}{v}} e^{i \operatorname{sign}(\vartheta)(1+\nu m) \pi / 2} .
$$

Lemma 2.4 Let $0<v \leq 1$ be noninteger, the left-hand side of $\mathcal{A B R}$ fractional derivative ${ }^{A B R} \mathcal{D}_{+}^{v}$ satisfies the following condition:

$$
\mathcal{A B R}^{\mathcal{B}} \mathcal{D}_{+}^{v}\left(e^{i \operatorname{sign} v|\vartheta|^{\frac{1}{v}} t}\right)=\frac{\mathcal{B}(v)}{1-v} \sum_{m=0}^{\infty}\left(\frac{-v}{1-v}\right)^{m} e^{i \operatorname{sign}(\vartheta)|\vartheta|^{\frac{1}{v} t}}|\vartheta|^{-m} e^{-i \operatorname{sign}(\vartheta)(v m) \pi / 2} .
$$

Proof

$$
\begin{aligned}
& { }^{\mathcal{A B R}} \mathcal{D}_{+}^{v}\left(e^{i \operatorname{sign} v|\vartheta|^{\frac{1}{v}} t}\right) \\
& \quad=\frac{\mathcal{B}(v)}{1-v} \frac{d}{d t} \int_{-\infty}^{t} e^{i \operatorname{sign} v|\vartheta|^{\frac{1}{v}}} E_{\nu}\left(\frac{-v}{1-v}(t-s)^{v}\right) d s
\end{aligned}
$$




$$
\begin{aligned}
& =\frac{\mathcal{B}(v)}{1-v} \sum_{m=0}^{\infty}\left(\frac{-v}{1-v}\right)^{m} \frac{1}{\Gamma(m v+1)} \frac{d}{d t} \int_{-\infty}^{t} e^{i \operatorname{sign} v|\vartheta|^{\frac{1}{v}}}(t-s)^{m v} d s \\
& =\frac{\mathcal{B}(v)}{1-v} \sum_{m=0}^{\infty}\left(\frac{-v}{1-v}\right)^{m} \frac{d}{d t}\left({ }^{\mathrm{RL}} \mathcal{I}_{+}^{v m+1}\left(e^{i \operatorname{sign}(\vartheta)|\vartheta|^{\frac{1}{v}} t}\right)\right) \\
& =\frac{\mathcal{B}(v)}{1-v} \sum_{m=0}^{\infty}\left(\frac{-v}{1-v}\right)^{m} \frac{d}{d t}\left(e^{i \operatorname{sign}(\vartheta)|\vartheta|^{\frac{1}{v} t}}|\vartheta|^{\frac{-(v m+1)}{v}} e^{-i \operatorname{sign}(\vartheta)(1+v m) \pi / 2}\right) \\
& =\frac{\mathcal{B}(v)}{1-v} \sum_{m=0}^{\infty}\left(\frac{-v}{1-v}\right)^{m} e^{-i \operatorname{sign}(\vartheta)(1+v m) \pi / 2}|\vartheta|^{\frac{-(\nu m+1)}{v}} i|\vartheta|^{\frac{1}{v}} e^{i \operatorname{sign}(\vartheta)|\vartheta|^{\frac{1}{v} t}} \\
& =\frac{\mathcal{B}(\nu)}{1-v} \sum_{m=0}^{\infty}\left(\frac{-v}{1-v}\right)^{m} e^{i \operatorname{sign}(\vartheta)|\vartheta|^{\frac{1}{v} t}}|\vartheta|^{-m}(\cos (\nu m) \pi / 2-i \operatorname{sign}(\vartheta) \sin (\nu m) \pi / 2) \\
& =\frac{\mathcal{B}(v)}{1-v} \sum_{m=0}^{\infty}\left(\frac{-v}{1-v}\right)^{m} e^{i \operatorname{sign}(\vartheta)|\vartheta|^{\frac{1}{v} t}}|\vartheta|^{-m} e^{-i \operatorname{sign}(\vartheta)(v m) \pi / 2} .
\end{aligned}
$$

This completes the proof.

The following lemma is derived in a similar way.

Lemma 2.5 Let $v \in(0,1)$, the right-hand side of $\mathcal{A B R}$ fractional derivative pertaining to function $\mathcal{U}(t)$ is formulated as follows:

$$
\mathcal{A B R}_{\mathcal{D}_{-}^{v}}\left(e^{i \operatorname{sign} v|\vartheta|^{\frac{1}{v}} t}\right)=\frac{\mathcal{B}(v)}{1-v} \sum_{m=0}^{\infty}(-1)^{m+1}\left(\frac{v}{1-v}\right)^{m} e^{i \operatorname{sign}(\vartheta)|\vartheta|^{\frac{1}{v} t}}|\vartheta|^{-m} e^{i \operatorname{sign}(\vartheta)(v m) \pi / 2}
$$

Some new operational relations are presented next. They are derived for a general fractional derivative operator $\mathcal{D}_{\beta}^{v}$, which contains both the left- and right-hand side pertaining to the $\mathcal{A B R}$ fractional derivatives:

$$
\left(\mathcal{D}_{\beta}^{v} \mathcal{U}\right)(t)=(1-\beta)\left(\mathcal{D}_{+}^{v} \mathcal{U}\right)(t)-\beta\left(\mathcal{D}_{-}^{v} \mathcal{U}\right)(t), \quad 0<v \leq 1, \beta \in \mathbb{R}
$$

Theorem 2.4 Given $0<v \leq 1$ and a mapping $\mathcal{U} \in \Phi(\mathbb{R})$, the fractional Fourier transform of fractional operator in equation (2.6) is expressed in the following form:

$$
\mathcal{F}_{\nu}\left({ }^{\mathcal{A B R}} \mathcal{D}_{\beta}^{\nu} \mathcal{U}(t)\right)(\vartheta)=\sum_{m=0}^{\infty} \mathcal{H}_{\nu}(\vartheta)|\vartheta|^{-m}\left(\mathcal{F}_{\nu} \mathcal{U}(t)\right)(\vartheta)
$$

where $\mathcal{H}_{v}(\vartheta)$ is given by

$$
\mathcal{H}_{v}(\vartheta)=\frac{\mathcal{B}(v)}{1-v}\left(\frac{-v}{1-v}\right)^{m}(-\cos (v m \pi / 2)+i \operatorname{sign}(\vartheta)(1-2 \beta) \sin (v m \pi / 2)) \text {. }
$$

Proof If $\vartheta=0$, we have $\mathcal{F}_{\nu}\left({ }^{\mathcal{B R} R} \mathcal{D}_{\beta}^{\nu} \mathcal{U}\right)(0)=0$ for any $\mathcal{U}$ that belongs to $\Phi(\mathbb{R})$.

For $\vartheta \neq 0$,

$$
\begin{aligned}
& \mathcal{F}_{\nu}\left({ }^{\mathcal{A B R}} \mathcal{D}_{\beta}^{\nu} \mathcal{U}(t)\right)(\vartheta) \\
& =\int_{-\infty}^{\infty} e^{i \operatorname{sign}(\vartheta)|\vartheta|^{\frac{1}{v}} t}\left(\mathcal{A B R} \mathcal{D}_{\beta}^{v} \mathcal{U}(t)\right) d t
\end{aligned}
$$




$$
\begin{aligned}
= & (1-\beta) \int_{-\infty}^{\infty} e^{i \operatorname{sign}(\vartheta)|\vartheta|^{\frac{1}{v}} t}\left(\mathcal{A B R} \mathcal{D}_{+}^{v} \mathcal{U}(t)\right) d t-\beta \int_{-\infty}^{\infty} e^{i \operatorname{sign}(\vartheta)|\vartheta|^{\frac{1}{v}} t}\left(\mathcal{A B R}^{v} \mathcal{D}_{-}^{v} \mathcal{U}(t)\right) d t \\
= & (1-\beta) \int_{-\infty}^{\infty}\left(\mathcal{A B R}^{v} \mathcal{D}_{-}^{v} e^{i \operatorname{sign} \vartheta|\vartheta|^{\frac{1}{v}} t}\right) \mathcal{U}(t) d t-\beta \int_{-\infty}^{\infty}\left(\mathcal{A B \mathcal { R }}^{v} \mathcal{D}_{+}^{v} e^{i \operatorname{sign} \vartheta|\vartheta|^{\frac{1}{v}} t}\right) \mathcal{U}(t) d t \\
= & (\beta-1) \frac{\mathcal{B}(v)}{1-v} \sum_{m=0}^{\infty}\left(\frac{-v}{1-v}\right)^{m}|\vartheta|^{-m} e^{-i \operatorname{sign}(\vartheta) v m \pi / 2} \int_{-\infty}^{\infty} e^{i \operatorname{sign} \vartheta|\vartheta|^{\frac{1}{v}}} \mathcal{U}(t) d t \\
& -(\beta) \frac{\mathcal{B}(v)}{1-v} \sum_{m=0}^{\infty}\left(\frac{-v}{1-v}\right)^{m}|\vartheta|^{-m} e^{i \operatorname{sign}(\vartheta) v m \pi / 2} \int_{-\infty}^{\infty} e^{i \operatorname{sign} \vartheta|\vartheta|^{\frac{1}{v}}} \mathcal{U}(t) d t \\
= & -\frac{\mathcal{B}(v)}{1-v} \sum_{m=0}^{\infty}\left(\frac{-v}{1-v}\right)^{m}|\vartheta|^{-m} \cos (v m \pi / 2) \mathcal{F}_{v}(\mathcal{U}(t)) \\
& +i(1-2 \beta) \operatorname{sign}(\vartheta) \frac{\mathcal{B}(v)}{1-v} \sum_{m=0}^{\infty}\left(\frac{-v}{1-v}\right)^{m}|\vartheta|^{-m} \sin (v m \pi / 2) \mathcal{F}_{v}(\mathcal{U}(t)) \\
= & \sum_{m=0}^{\infty} \mathcal{H}_{v}(\vartheta)|\vartheta|^{-m} \mathcal{F}_{v}(\mathcal{U}(t))(\vartheta),
\end{aligned}
$$

where $\mathcal{H}_{v}(\vartheta)=\frac{\mathcal{B}(\nu)}{1-\nu}\left(\frac{-v}{1-\nu}\right)^{m}(-\cos (\nu m \pi / 2)+i(1-2 \beta) \operatorname{sign}(\vartheta) \sin (\nu m \pi / 2))$.

Remark 2.1 Let us take $\mathcal{H}_{\nu_{1}}(\vartheta)=\frac{\mathcal{B}(v)}{1-\nu}\left(\frac{-v}{1-\nu}\right)^{m}(-\cos (v m \pi / 2)-i(1-2 \beta) \sin (\nu m \pi / 2))$ for $\vartheta<0$ and $\mathcal{H}_{\nu_{2}}(\vartheta)=\frac{\mathcal{B}(\nu)}{1-\nu}\left(\frac{-v}{1-\nu}\right)^{m}(-\cos (\nu m \pi / 2)+i(1-2 \beta) \sin (\nu m \pi / 2))$ for $\vartheta \geq 0$.

Definition 2.9 FODE

$$
\left({ }^{\mathcal{A B R}} \mathcal{D}_{\beta}^{v} \mathcal{U}\right)(t)=\mathcal{G}(t), \quad \forall t \in \mathbb{R}
$$

is stable in the Hyers-Ulam sense if there exists a continuously differentiable mapping $\mathcal{U}: \mathbb{R} \rightarrow \mathbb{F}$ that is able to satisfy the following inequality:

$$
\left|\left({ }^{\mathcal{A B R}} \mathcal{D}_{\beta}^{v} \mathcal{U}(t)\right)-\mathcal{G}(t)\right| \leq \epsilon, \quad \forall t \in \mathbb{R}
$$

there exists a solution $\mathcal{U}: \mathbb{R} \rightarrow \mathbb{F}$ of differential equation (2.8) with

$$
\left|\mathcal{U}(t)-\mathcal{U}_{v}(t)\right| \leq K \epsilon, \quad \forall t \in \mathbb{R},
$$

where $\epsilon>0$ and $K>0$ is Hyers-Ulam stability(HUS) constant.

Definition 2.10 FODE (2.8) is stable in the generalized Hyers-Ulam sense if there exists a continuously differentiable mapping $\phi: \mathbb{R} \rightarrow \mathbb{R}$ in a way that given any solution $\mathcal{U}: \mathbb{R} \rightarrow \mathbb{F}$ that is able to satisfy inequality (2.9), a solution $\mathcal{U}_{v}: \mathbb{R} \rightarrow \mathbb{F}$ of the considered equation (2.8) is available, in which

$$
\left|\mathcal{U}(t)-\mathcal{U}_{v}(t)\right| \leq \phi(\epsilon), \quad \forall t \in \mathbb{R} .
$$

Definition 2.11 FODE (2.8) is stable in the Hyers-Ulam-Rassias sense subject to $\phi: \mathbb{R} \rightarrow$ $\mathbb{R}$ if there exists $K_{\phi} \in \mathbb{R}$, in a way that given any $\epsilon>0$ and any solution $\mathcal{U}: \mathbb{R} \rightarrow \mathbb{F}$ that is 
able to satisfy the following inequality:

$$
\left|\left({ }^{\mathcal{A B R}} \mathcal{D}_{\beta}^{\nu} \mathcal{U}\right)(t)-\mathcal{G}(t)\right| \leq \epsilon \phi(t), \quad \forall t \in \mathbb{R}
$$

a unique solution $\mathcal{U}_{\nu}: \mathbb{R} \rightarrow \mathbb{F}$ with respect to considered problem (2.8) exists, in which

$$
\left|\mathcal{U}(t)-\mathcal{U}_{v}(t)\right| \leq K_{\phi} \epsilon \phi(t), \quad \forall t \in \mathbb{R}
$$

Definition 2.12 Fractional differential equation (2.8) is stable in the generalized HyersUlam-Rassias sense subject to $\phi: \mathbb{R} \rightarrow \mathbb{R}$ if there exists $K_{\phi} \in \mathbb{R}$, in such a way that given any solution $\mathcal{U}: \mathbb{R} \rightarrow \mathbb{F}$ that is able to satisfy the following inequality:

$$
\left|\left({ }^{\mathcal{A B R}} \mathcal{D}_{\beta}^{v} \mathcal{U}\right)(t)-\mathcal{G}(t)\right| \leq \phi(t), \quad \forall t \in \mathbb{R},
$$

a unique solution $\mathcal{U}_{\nu}: \mathbb{R} \rightarrow \mathbb{F}$ of differential equation (2.8) exists, in which

$$
\left|\mathcal{U}(t)-\mathcal{U}_{\nu}(t)\right| \leq K_{\phi} \phi(t), \quad \forall t \in \mathbb{R}
$$

\section{Analysis of the Hyers-Ulam stability for linear equations}

The results related to Hyers-Ulam stability of FODE are derived by using the fractional Fourier transform as follows:

$$
\left({ }^{\mathcal{A B R}} \mathcal{D}_{\beta}^{v} \mathcal{U}\right)(t)=\mathcal{G}(t), \quad t \in \mathbb{R}
$$

Theorem 3.1 Suppose that $0<v \leq 1$ and a given real continuous function in $\Phi(\mathbb{R})$ is denoted by $\mathcal{G}(t)$. Suppose that a function $\mathcal{U}: \mathbb{R} \rightarrow \mathbb{F}$ that is able to satisfy the following inequality:

$$
\left|\left({ }^{\mathcal{A B R}} \mathcal{D}_{\beta}^{v} \mathcal{U}\right)(t)-\mathcal{G}(t)\right| \leq \epsilon
$$

for all $t \in \mathbb{R}$ and for some $\epsilon>0$, a solution $\mathcal{U}_{v}: \mathbb{R} \rightarrow \mathbb{F}$ of FODE (3.1) exists, in which

$$
\left|\mathcal{U}(t)-\mathcal{U}_{v}(t)\right| \leq K \epsilon
$$

Proof A function $\mathcal{Y}_{1}: \mathbb{R} \rightarrow \mathbb{F}$ is defined in which

$$
\mathcal{Y}_{1}(t)=\left({ }^{\mathcal{A B R}} D_{\beta}^{\nu} \mathcal{U}\right)(t)-\mathcal{G}(t)
$$

Suppose that $\mathcal{G}(t)$ is a continuously differentiable function satisfying inequality (3.2), then $\left|\mathcal{Y}_{1}(t)\right| \leq \epsilon$ for each $\epsilon>0$. By imposing the fractional Fourier transform operator $\mathcal{F}_{\nu}$ onto both sides of equation (3.3), we have

$$
\begin{aligned}
\mathcal{F}_{\nu}\left(\mathcal{Y}_{1}(t)\right)(\vartheta) & =\mathcal{F}_{\nu}\left(\left(\mathcal{A B R}^{\nu} D_{\beta}^{v} \mathcal{U}(t)\right)(\vartheta)\right)-\mathcal{F}_{\nu}(\mathcal{G}(t))(\vartheta) \\
& =\sum_{m=0}^{\infty} \mathcal{H}_{v}(\vartheta)|\vartheta|^{-m} \mathcal{F}_{\nu}(\mathcal{U}(t))(\vartheta)-\mathcal{F}_{v}(\mathcal{G}(t))(\vartheta)
\end{aligned}
$$




$$
\begin{aligned}
\mathcal{F}_{\nu}(\mathcal{U}(t))(\vartheta)= & \frac{1}{\sum_{m=0}^{\infty} \mathcal{H}_{\nu}(\vartheta)|\vartheta|^{-m}}\left(\mathcal{F}_{\nu} \mathcal{G}(t)\right)(\vartheta) \\
& +\frac{1}{\sum_{m=0}^{\infty} \mathcal{H}_{v}(\vartheta)|\vartheta|^{-m}} \mathcal{F}_{\nu}\left(\mathcal{Y}_{1}(t)\right)(\vartheta)
\end{aligned}
$$

Set

$$
\begin{aligned}
\mathcal{U}_{\nu}(t)= & \frac{1}{2 \pi} \sum_{m=0}^{\infty} \Gamma(1+v m) \int_{-\infty}^{\infty}\left(\mathcal{H}_{1} \cos (v m \pi / 2)+i \mathcal{H}_{2} \operatorname{sign}(t-\tau)\right. \\
& \times \sin (v m \pi / 2)) \operatorname{sign}(t-\tau)|t-\tau|^{-1-v m} \mathcal{G}(\tau) d \tau,
\end{aligned}
$$

where $\mathcal{H}_{1}=\frac{-i}{\mathcal{H}_{v_{1}}}+\frac{i}{\mathcal{H}_{v_{2}}}$ and $\mathcal{H}_{2}=\frac{i}{\mathcal{H}_{v_{1}}}+\frac{i}{\mathcal{H}_{v_{2}}}$. By the definition of convolution, we have

$$
\begin{aligned}
\mathcal{U}_{\nu}(t)= & \frac{1}{2 \pi} \sum_{m=0}^{\infty} \operatorname{sign}(t)|t|^{-1-v m} \Gamma(1+v m)\left(\mathcal{H}_{1} \cos (v m \pi / 2)+i \operatorname{sign}(t) \mathcal{H}_{2} \sin (\nu m \pi / 2)\right) \\
& * \mathcal{G}(t) \\
= & \frac{1}{2 \pi}\left(\sum_{m=0}^{\infty} \frac{1}{\mathcal{H}_{\nu_{1}}} \int_{0}^{\infty} e^{i \xi t} \xi^{(1+v m)-1} d \xi+\sum_{m=0}^{\infty} \frac{1}{\mathcal{H}_{\nu_{2}}} \int_{0}^{\infty} e^{-i \xi t} \xi^{(1+v m)-1} d \xi\right) * \mathcal{G}(t) \\
= & \frac{1}{2 \pi}\left(\sum_{m=0}^{\infty} \frac{1}{\mathcal{H}_{v_{1}}} \int_{0}^{\infty} e^{i \xi t} \xi^{(1+v m)-v} \xi^{\nu-1} d \xi+\sum_{m=0}^{\infty} \frac{1}{\mathcal{H}_{v_{2}}} \int_{0}^{\infty} e^{-i \xi t} \xi^{(1+v m)-v} \xi^{\nu-1} d \xi\right) \\
& * \mathcal{G}(t) .
\end{aligned}
$$

By using substitution $\xi^{\nu}=|\vartheta|, \xi=|\vartheta|^{\frac{1}{v}}$ and $d \vartheta=\nu \xi^{\nu-1} d \xi$,

$$
\begin{aligned}
& \mathcal{U}_{\nu}(t)= \frac{1}{2 \pi v}\left(\sum_{m=0}^{\infty} \frac{1}{\mathcal{H}_{\nu_{1}}} \int_{-\infty}^{0} e^{i(-\vartheta)^{\frac{1}{v}} t}(-\vartheta)^{\frac{1}{v}+m-1} d \vartheta\right. \\
&\left.+\sum_{m=0}^{\infty} \frac{1}{\mathcal{H}_{v_{2}}} \int_{0}^{\infty} e^{i(\vartheta)^{\frac{1}{v}} t}(\vartheta)^{\frac{1}{v}+m-1} d \vartheta\right) * \mathcal{G}(t) \\
&= \frac{1}{2 \pi v}\left(\int_{-\infty}^{\infty} \sum_{m=0}^{\infty}\left(\frac{1}{\mathcal{H}_{\nu}(\vartheta)|\vartheta|^{-m}}\right) e^{-i \operatorname{sign}(\vartheta)|\vartheta|^{\frac{1}{v}}}|\vartheta|^{\frac{1}{v}-1} d \vartheta\right) * \mathcal{G}(t) \\
&= \mathcal{F}_{\nu}^{-1}\left(\frac{1}{\sum_{m=0}^{\infty} \mathcal{H}_{\nu}(\vartheta)|\vartheta|^{-m}}\right) * \mathcal{G}(t), \\
&\left(\mathcal{F}_{\nu} \mathcal{U}_{\nu}(t)\right)(\vartheta)=\frac{1}{\sum_{m=0}^{\infty} \mathcal{H}_{\nu}(\vartheta)|\vartheta|^{-m}}\left(\mathcal{F}_{\nu} \mathcal{G}(t)\right)(\vartheta) .
\end{aligned}
$$

By equations (2.7), (3.6) and simple computation, we obtain

$$
\begin{aligned}
\mathcal{F}_{\nu}\left({ }^{\mathcal{A B R}} \mathcal{D}_{\beta}^{v} \mathcal{U}_{v}(t)\right)(\vartheta) & =\sum_{m=0}^{\infty} \mathcal{H}_{v}(\vartheta)|\vartheta|^{-m}\left(\mathcal{F}_{\nu} \mathcal{U}_{v}\right)(\vartheta) \\
& =\sum_{m=0}^{\infty} \mathcal{H}_{\nu}(\vartheta)|\vartheta|^{-m} \frac{1}{\sum_{m=0}^{\infty} \mathcal{H}_{\nu}(\vartheta)|\vartheta|^{-m}}\left(\mathcal{F}_{\nu} \mathcal{G}\right)(\vartheta), \\
\mathcal{F}_{\nu}\left({ }^{\mathcal{A B R}} \mathcal{D}_{\beta}^{v} \mathcal{U}_{\nu}(t)\right)(\vartheta) & =\left(\mathcal{F}_{\nu} \mathcal{U}(t)\right)(\vartheta) .
\end{aligned}
$$


Since $\mathcal{F}_{v}$ is one-to-one, it follows that ${ }^{\mathcal{A B R}} \mathcal{D}_{\beta}^{v} \mathcal{U}_{v}(t)=\mathcal{G}(t)$, so $\mathcal{U}_{v}(t)$ is a solution of equation (3.1). In view of equations (3.4) and (3.6), we obtain

$$
\left(\mathcal{F}_{\nu} \mathcal{U}(t)\right)(\vartheta)-\left(\mathcal{F}_{\nu} \mathcal{U}_{\nu}(t)\right)(\vartheta)=\frac{1}{\sum_{m=0}^{\infty} \mathcal{H}_{\nu}(\vartheta)|\vartheta|^{-m}} \mathcal{F}_{\nu}\left(\mathcal{Y}_{1}(t)\right)(\vartheta)
$$

By the convolution property of Fourier transform, we get

$$
\begin{aligned}
\mathcal{U}(t)-\mathcal{U}_{v}(t)= & \mathcal{F}_{\nu}^{-1}\left(\frac{1}{\sum_{m=0}^{\infty} \mathcal{H}_{v}(\vartheta)|\vartheta|^{-m}}\right) * \mathcal{Y}_{1}(t) \\
= & \frac{1}{2 \pi v}\left(\int_{-\infty}^{\infty} \frac{1}{\sum_{m=0}^{\infty} \mathcal{H}_{v}(\vartheta)|\vartheta|^{-m}} e^{-i \operatorname{sign}(\vartheta)|\vartheta|^{\frac{1}{v}} t}|\vartheta|^{\frac{1}{v}-1} d \vartheta\right) * \mathcal{Y}_{1}(t) \\
= & \frac{1}{2 \pi v} \sum_{m=0}^{\infty} \frac{1}{\mathcal{H}_{v_{1}}} \int_{-\infty}^{0}(-\vartheta)^{m} e^{i(-\vartheta)^{\frac{1}{v}} t}(-\vartheta)^{\frac{1}{v}-1} d \vartheta * \mathcal{Y}_{1}(t) \\
& +\frac{1}{2 \pi v} \sum_{m=0}^{\infty} \frac{1}{\mathcal{H}_{v_{2}}} \int_{0}^{\infty}(\vartheta)^{m} e^{i(\vartheta)^{\frac{1}{v}} t}(\vartheta)^{\frac{1}{v}-1} d \vartheta * \mathcal{Y}_{1}(t) \\
= & \frac{1}{2 \pi}\left(\sum_{m=0}^{\infty} \frac{1}{\mathcal{H}_{v_{1}}} \int_{0}^{\infty} e^{-i \xi t} \xi^{v m} d z+\sum_{m=0}^{\infty} \frac{1}{\mathcal{H}_{v_{2}}} \int_{0}^{\infty} e^{i \xi t} \xi^{\nu m} d \xi\right) * \mathcal{Y}_{1}(t) \\
= & \frac{1}{2 \pi} \sum_{m=0}^{\infty} \Gamma(1+v m) \operatorname{sign}(t)|t|^{-1-v m}\left(\frac{1}{\mathcal{H}_{v_{1}}}(-i \cos (v m \pi / 2)-\operatorname{sign}(t)\right. \\
& \times \sin (v m \pi / 2))+\frac{1}{\mathcal{H}_{\nu_{2}}}(i \cos (v m \pi / 2)-\operatorname{sign}(t) \sin (v m \pi / 2)) * \mathcal{Y}_{1}(t) \\
= & \frac{1}{2 \pi} \sum_{m=0}^{\infty} \Gamma(1+v m) \int_{\mathbb{R}}\left(\mathcal{H}_{1} \cos (v m \pi / 2)\right. \\
& \left.+i \operatorname{sign}(t-\tau) \mathcal{H}_{2} \sin (v m \pi / 2)\right) \operatorname{sign}(t-\tau)|t-\tau|^{-1-v m} \mathcal{Y}_{1}(t) .
\end{aligned}
$$

Now, by imposing modulus on both sides of equation (3.7), we obtain

$$
\begin{aligned}
& \left|\mathcal{U}(t)-\mathcal{U}_{v}(t)\right| \leq C\left|\int_{\mathbb{R}}(t-\tau)^{-1-v m} \mathcal{Y}_{1}(\tau) d \tau\right| \\
& \left|\mathcal{U}(t)-\mathcal{U}_{v}(t)\right| \leq C \epsilon \int_{\mathbb{R}}(t-\tau)^{-1-v m} d \tau, \\
& \left|\mathcal{U}(t)-\mathcal{U}_{v}(t)\right| \leq K \epsilon,
\end{aligned}
$$

where $C=\left|\mathcal{H}_{1} \cos (v m \pi / 2)+i \operatorname{sign}(t-\tau) \mathcal{H}_{2} \sin (v m \pi / 2)\right| \in \mathbb{R}[52]$ and $K=C \int_{\mathbb{R}}(t-$ $\tau)^{-1-v m} d \tau$ for any values of $t$. Hence FODE (3.1) is stable in the sense of Hyers-Ulam. The proof is completed.

Remark 3.1 Putting $\phi(\epsilon)=K \epsilon$ yields that FODE (3.1) is stable in the generalized HyersUlam sense.

FODE (3.1) is stable in the Hyers-Ulam-Rassias sense can be examined in a similar manner. 
Corollary 3.1 Suppose $0<v \leq 1$ and a real continuous function on $\mathbb{R}$ is denoted by $\mathcal{G}(t)$. A continuous function $\phi(t)$ exists, in which $\mathcal{U}: \mathbb{R} \rightarrow \mathbb{F}$ is a continuously differentiable function that is able to satisfy the following inequality:

$$
\left|\left({ }^{\mathcal{A B R}} \mathcal{D}_{\beta}^{\nu} \mathcal{U}\right)(t)-\mathcal{G}(t)\right| \leq \epsilon \phi(t)
$$

for all $t \in \mathbb{R}$. As a result, solution $\mathcal{U}_{v}: \mathbb{R} \rightarrow \mathbb{F}$ with respect to $F O D E$ (3.1) exists, in which

$$
\left|\mathcal{U}(t)-\mathcal{U}_{\nu}(t)\right| \leq K \epsilon \phi(t), \quad \forall t \in \mathbb{R}
$$

Remark 3.2 If we put $\epsilon=1$ in inequality (3.8), then by definition the considered problem is stable in the generalized Hyers-Ulam-Rassias sense.

\section{Analysis of the existence and stability results for nonlinear equation}

The existence and Hyers-Ulam-Rassias stability with respect to nonlinear FODE is analyzed as follows:

$$
{ }^{\mathcal{A B R}} \mathcal{D}_{\beta}^{v} \mathcal{U}(t)=\mathcal{G}(t, \mathcal{U}(t)), \quad \forall t \in \mathbb{R}
$$

The following hypotheses are introduced:

$1\left[\mathcal{D}_{1}\right] \mathcal{U}:[-T, T] X \mathbb{R} \rightarrow \mathbb{F}$ is continuous;

$2\left[\mathcal{D}_{2}\right]$ For $t \in[-T, T]$, there exists a constant $0<\mathbb{L}<1$ such that

$$
\left|\mathcal{G}\left(t, \mathcal{U}_{1}\right)-\mathcal{G}\left(t, \mathcal{U}_{2}\right)\right| \leq \mathbb{L}\left|\mathcal{U}_{1}-\mathcal{U}_{2}\right|, \quad \forall \mathcal{U}_{1}, \mathcal{U}_{2} \in \mathbb{R}
$$

$3\left[\mathcal{D}_{3}\right]$ There exists a constant $\mathbb{L}_{\mathcal{U}}>0$ such that

$$
|\mathcal{G}(t, \mathcal{U})| \leq \mathbb{L}_{\mathcal{U}}(1+|\mathcal{U}|), \quad \forall \mathcal{U} \in \mathbb{R}
$$

Suppose that the space $\psi=\mathcal{C}(\mathbb{R}, \mathbb{F})$ has the following defined norm:

$$
\|\mathcal{U}\|=\sup \{|\mathcal{U}(t)|: t \in \mathbb{R}\}
$$

Theorem 4.1 Suppose that hypotheses $\left[\mathcal{D}_{1}\right]$ and $\left[\mathcal{D}_{2}\right]$ hold. If $\mathbb{L} C \frac{(2 T)^{-v m}}{v m}<1$, then equation (4.1) has a unique solution in $\psi$.

Proof Define an operator $\Delta: \psi \rightarrow \psi$ as follows:

$$
\begin{aligned}
(\Delta \mathcal{U})(t)= & \frac{1}{2 \pi} \sum_{m=0}^{\infty} \Gamma(1+\nu m) \int_{-t}^{t}\left(\mathcal{H}_{1} \cos (\nu m \pi / 2)+i \mathcal{H}_{2} \operatorname{sign}(t-\tau) \sin (\nu m \pi / 2)\right) \\
& \times|t-\tau|^{-1-v m} \mathcal{G}(\tau, \mathcal{U}(\tau)) d \tau
\end{aligned}
$$

for any $t \in[-T, T]$. Due to $\Delta$ being well defined, suppose $\mathcal{U}_{1}, \mathcal{U}_{2} \in \psi$ and given $t \in[-T, T]$, we have

$$
\left|\left(\Delta \mathcal{U}_{1}\right)(t)-\left(\Delta \mathcal{U}_{2}\right)(t)\right| \leq \int_{-t}^{t}\left|\left(\mathcal{H}_{1} \cos (\nu m \pi / 2)+i \mathcal{H}_{2} \operatorname{sign}(t-\tau) \sin (\nu m \pi / 2)\right)\right|
$$




$$
\begin{aligned}
& \times|t-\tau|^{-1-v m}\left|\mathcal{G}\left(\tau, \mathcal{U}_{1}(\tau)\right)-\mathcal{G}\left(\tau, \mathcal{U}_{2}(\tau)\right)\right| d \tau \\
\leq & \mathbb{L} C \int_{-t}^{t}|t-\tau|^{-1-v m}\left|\mathcal{U}_{1}-\mathcal{U}_{2}\right| d \tau \\
\leq & \mathbb{L} C \frac{(2 T)^{-v m}}{\nu m}\left\|\mathcal{U}_{1}-\mathcal{U}_{2}\right\|,
\end{aligned}
$$

where $C=\left|\mathcal{H}_{1} \cos (v m \pi / 2)+i \operatorname{sign}(t-\tau) \mathcal{H}_{2} \sin (v m \pi / 2)\right| \in \mathbb{R}$ [52]. From the condition $\mathbb{L} C \frac{(2 T)^{-v m}}{\nu m}<1, \Delta$ takes the form of a contraction mapping. As a result, in accordance with the Banach contraction principle, $\Delta$ has a unique fixed point that forms a unique solution of FODE (4.1).

Theorem 4.2 Operator $\Delta$ is compact based on hypotheses $\left[\mathcal{D}_{1}\right]-\left[\mathcal{D}_{3}\right]$.

Proof Consider the definition in equation (4.2) for the operator $\Delta$. The proof is divided into several steps.

Step (1): $\Delta$ is continuous

Suppose that $\mathcal{U}_{n}$ such that $\mathcal{U}_{n} \rightarrow \mathcal{U}$ in $\psi$. As such, given all $t \in[-T, T]$, we have

$$
\left|\left(\Delta \mathcal{U}_{n}\right)(t)-(\Delta \mathcal{U})(t)\right| \leq C \int_{-t}^{t}|t-\tau|^{-1-\nu m}\left|\mathcal{G}\left(\tau, \mathcal{U}_{n}(\tau)\right)-\mathcal{G}(\tau, \mathcal{U}(\tau))\right| d \tau
$$

Based on $\left[\mathcal{D}_{2}\right]$, we can have

$$
\left|\mathcal{G}\left(t, \mathcal{U}_{n}\right)-\mathcal{G}(t, \mathcal{U})\right| \leq \mathbb{L}\left|\mathcal{U}_{n}-\mathcal{U}\right|
$$

As such, we obtain

$$
\begin{aligned}
\left|\left(\Delta \mathcal{U}_{n}\right)(t)-(\Delta \mathcal{U})(t)\right| & \leq \mathbb{L} C \int_{-t}^{t}|t-\tau|^{-1-v m}\left|\mathcal{U}_{n}-\mathcal{U}\right| d \tau \\
& \leq \mathbb{L} C \frac{(2 T)^{-v m}}{\nu m}\left|\mathcal{U}_{n}-\mathcal{U}\right|
\end{aligned}
$$

As we assume $\mathcal{U}_{n} \rightarrow \mathcal{U}$ as $n \rightarrow \infty$ for every $t \in[-T, T]$, the following can be obtained in accordance with the Lebesgue dominated convergence theorem [53]:

$$
\left|\left(\Delta \mathcal{U}_{n}\right)(t)-(\Delta \mathcal{U})(t)\right| \rightarrow 0 \quad \text { as } n \rightarrow \infty,
$$

hence

$$
\left\|\left(\Delta \mathcal{U}_{n}\right)(t)-(\Delta \mathcal{U})(t)\right\| \rightarrow 0 \quad \text { as } n \rightarrow \infty .
$$

This shows that $\Delta$ is continuous.

Step (2): We establish that $\Delta$ maps a bounded set in $\psi$. For this we just need to prove that, for any $\kappa^{*}>0$, there exists $\rho>0$, in which for any

$$
\mathcal{U} \in E^{*}=\left\{\mathcal{U} \in \psi:\|\mathcal{U}\| \leq \kappa^{*}\right\}
$$


we have

$$
\|\Delta(\mathcal{U})\| \leq \rho .
$$

In fact, for any $t \in[-T, T]$, from equation (4.2), we have

$$
\begin{aligned}
|(\Delta \mathcal{U}(t))|= & \frac{1}{2 \pi} \sum_{m=0}^{\infty} \Gamma(1+v m) \int_{-t}^{t}\left|\left(\mathcal{H}_{1} \cos (v m \pi / 2)+i \mathcal{H}_{2} \operatorname{sign}(t-\tau) \sin (v m \pi / 2)\right)\right| \\
& \times \operatorname{sign}(t-\tau)|t-\tau|^{-1-v m}|\mathcal{G}(\tau, \mathcal{U}(\tau))| d \tau
\end{aligned}
$$

where $\mathcal{G} \in \psi$. Based on $\left[\mathcal{D}_{3}\right]$, we have

$$
\begin{aligned}
|(\Delta \mathcal{U}(t))| & \leq C \int_{-t}^{t}|t-\tau|^{-1-v m}|\mathcal{G}(\tau, \mathcal{H}(\tau))| d \tau \\
& \leq C \mathbb{L}_{\mathcal{U}}(1+\rho) \int_{-t}^{t}|t-\tau|^{-1-v m} d \tau .
\end{aligned}
$$

As a result, we have

$$
|(\Delta \mathcal{U}(t))| \leq C \mathbb{L}_{\mathcal{U}}(1+\rho) \frac{(2 T)^{-v m}}{\nu m}=K
$$

Hence $\Delta\left(E^{*}\right)$ is bounded.

Step (3): We prove that the operator $\Delta$ is equicontinuous in $\psi$. Suppose $t_{1}, t_{2} \in[-T, T]$ with $0 \leq t_{1} \leq t_{2} \leq T$, as $E^{*}$ is a bounded set in $\psi$, and assume $\mathcal{U} \in E^{*}$. As such,

$$
\begin{aligned}
& \left|\left(\Delta \mathcal{U}\left(t_{1}\right)\right)-\left(\Delta \mathcal{U}\left(t_{2}\right)\right)\right| \\
& =\frac{1}{2 \pi} \sum_{m=0}^{\infty} \Gamma(1+v m) \\
& \quad \times \int_{-t_{1}}^{t_{1}}\left|\left(\mathcal{H}_{1} \cos (v m \pi / 2)+i \mathcal{H}_{2} \operatorname{sign}\left(t_{1}-\tau\right) \sin (v m \pi / 2)\right)\right|\left|t_{1}-\tau\right|^{-1-v m} \\
& \quad \times|\mathcal{G}(\tau, \mathcal{U}(\tau))| d \tau-\frac{1}{2 \pi} \sum_{m=0}^{\infty} \Gamma(1+v m) \\
& \quad \times \int_{-t_{2}}^{t_{2}}\left|\left(\mathcal{H}_{1} \cos (v m \pi / 2)-i \mathcal{H}_{2} \operatorname{sign}\left(t_{2}-\tau\right) \sin (v m \pi / 2)\right)\right| \\
& \quad \times\left|t_{2}-\tau\right|^{-1-v m}|\mathcal{G}(\tau, \mathcal{U}(\tau))| d \tau .
\end{aligned}
$$

Based on $\left[\mathcal{D}_{3}\right]$, we have

$$
\begin{aligned}
& \left|\left(\Delta \mathcal{U}\left(t_{1}\right)\right)-\left(\Delta \mathcal{U}\left(t_{2}\right)\right)\right| \\
& \quad \leq \sum_{m=0}^{\infty} \frac{\Gamma(1+v m)}{2 \pi} C \mathbb{L}_{\mathcal{U}}(1+\rho)\left(\int_{-t_{1}}^{t_{1}}\left(\left|t_{1}-\tau\right|^{-1-v m}-\left|t_{2}-\tau\right|^{-1-v m}\right) d \tau\right. \\
& \left.\quad+\int_{-t_{2}}^{-t_{1}}\left|t_{2}-\tau\right|^{-1-v m} d \tau+\int_{t_{1}}^{t_{2}}\left|t_{2}-\tau\right|^{-1-v m} d \tau\right)
\end{aligned}
$$




$$
\begin{aligned}
& \times\left|\left(\Delta \mathcal{U}\left(t_{1}\right)\right)-\left(\Delta \mathcal{U}\left(t_{2}\right)\right)\right| \\
\leq & \sum_{m=0}^{\infty} \frac{\Gamma(1+v m)}{2 \pi} C \mathbb{L}_{\mathcal{U}}(1+\rho) \\
& \times\left(-\frac{\left(2 t_{1}\right)^{-v m}}{\nu m}-\frac{\left(2 t_{2}\right)^{-v m}}{\nu m}-\frac{2\left(t_{2}-t_{1}\right)^{-v m}}{\nu m}+\frac{2\left(t_{1}+t_{2}\right)^{-v m}}{\nu m}\right) .
\end{aligned}
$$

Note that as $t_{1} \rightarrow t_{2}$, the right-hand side of the above inequality approximates zero, therefore $\Delta$ is equicontinuous. Based on steps (1) to (3), it is concluded that $\Delta$ is completely continuous. As such, operator $\Delta$ is compact in accordance with the Arzela-Ascoli theorem.

Next, we show the existence of solutions for equation (4.1) via Schaefer's fixed point theorem.

Theorem 4.3 Suppose that hypothesis $\left[\mathcal{D}_{3}\right]$ holds. If $C<1$, then $F O D E(4.1)$ has at least one solution in $\psi$.

Proof Now, a set $\mathcal{B} \subset \psi$ is considered. Its definition is in the form

$$
\mathcal{B}=\{\mathcal{U} \in \psi: \mathcal{U}=\eta \Delta \mathcal{U}, 0<\eta<1\}
$$

Let $\mathcal{U} \in \mathcal{B}$, in which

$$
\mathcal{U}(t)=\eta \Delta \mathcal{U}(t), \quad \eta \in(0,1)
$$

As a result, given any $t \in[-T, T]$, we obtain

$$
\begin{aligned}
\mathcal{U}(t)= & \eta \frac{1}{2 \pi} \sum_{m=0}^{\infty} \Gamma(1+\nu m) \int_{-t}^{t}\left(\mathcal{H}_{1} \cos (\nu m \pi / 2)+i \mathcal{H}_{2} \operatorname{sign}(t-\tau) \sin (\nu m \pi / 2)\right) \\
& \times \operatorname{sign}(t-\tau)|t-\tau|^{-1-v m} \mathcal{G}(\tau, \mathcal{U}(\tau)) d \tau .
\end{aligned}
$$

By taking maximum on both sides, we have

$$
\begin{aligned}
|\mathcal{U}(t)| & \leq C \int_{-t}^{t}|t-\tau|^{-1-v m}|\mathcal{G}(\tau, \mathcal{U}(\tau))| d \tau \\
\|\mathcal{U}(t)\| & \leq C \mathbb{L}_{\mathcal{U}} \int_{-t}^{t}|t-\tau|^{-1-v m}|1+\mathcal{U}(\tau)| d \tau \\
\|\mathcal{U}(t)\| & \leq C \mathbb{L}_{\mathcal{U}} \int_{-t}^{t}|t-\tau|^{-1-v m} d \tau+C \mathbb{L}_{\mathcal{U}} \int_{-t}^{t}|t-\tau|^{-1-v m}|\mathcal{U}(\tau)| d \tau \\
\|\mathcal{U}(t)\| & \leq C \mathbb{L}_{\mathcal{U}} \frac{(2 T)^{-v m}}{\nu m}+C \mathbb{L}_{\mathcal{U}} \frac{(2 T)^{-v m}}{\nu m}\|\mathcal{U}(t)\| .
\end{aligned}
$$

For simplicity, let

$$
N=C \mathbb{L}_{\mathcal{U}} \frac{(2 T)^{-v m}}{v m} .
$$


So, (4.3) becomes

$$
\begin{aligned}
\|\mathcal{U}(t)\| & \leq N+N\|\mathcal{U}(t)\|_{\mathcal{C}}, \\
\|\mathcal{U}(t)\| & \leq \frac{N}{1-N} .
\end{aligned}
$$

As such, $\mathcal{B}$ is bounded. According to Theorems 2.2 and 4.2, the operator $\Delta$ has at least one fixed point. Therefore, FODE (4.1) has at least one solution in $\psi$.

The following inequality is used for further analysis:

$$
\left|\left({ }^{\mathcal{A B R}} \mathcal{D}_{\beta}^{\nu} \mathcal{U}\right)(t)-\mathcal{G}(t, \mathcal{U}(t))\right| \leq G(t)
$$

We examine the generalized Hyers-Ulam stability through the following condition. [ $\left.\mathcal{D}_{4}\right]$ : Suppose that $\mathcal{G} \in \psi$ is a function and there exists $K_{\lambda}>0$ such that

$$
\begin{aligned}
& \int_{-t}^{t}\left(\mathcal{H}_{1} \cos (\nu m \pi / 2)+i \mathcal{H}_{2} \operatorname{sign}(t-\tau) \sin (\nu m \pi / 2)\right) \operatorname{sign}(t-\tau)|t-\tau|^{-1-\nu m} \mathcal{G}(\tau) d \tau \\
& \quad \leq K_{\lambda} G(t) .
\end{aligned}
$$

Theorem 4.4 Suppose that hypotheses $\left[\mathcal{D}_{1}\right],\left[\mathcal{D}_{2}\right]$, and $\left[\mathcal{D}_{4}\right]$ hold. If $\frac{(2 T)^{-v m}}{v m}<1$, then equation (4.1) is stable in the sense of Hyers-Ulam-Rassias with respect to G.

Proof A unique solution with respect to FODE (4.1) exists, i.e.,

$$
\begin{aligned}
\mathcal{Q}(t)= & \frac{1}{2 \pi} \sum_{m=0}^{\infty} \Gamma(1+\nu m) \int_{-t}^{t}\left(\mathcal{H}_{1} \cos (\nu m \pi / 2)+i \mathcal{H}_{2} \operatorname{sign}(t-\tau) \sin (\nu m \pi / 2)\right) \\
& \times \operatorname{sign}(t-\tau)|t-\tau|^{-1-\nu m} \mathcal{G}(\tau, \mathcal{Q}(\tau)) d \tau .
\end{aligned}
$$

Integrating inequality (4.4) from $-t$ to $t$ and using $\left[\mathcal{D}_{4}\right]$, we obtain

$$
\begin{aligned}
\mid \mathcal{U}(t) & -\frac{1}{2 \pi} \sum_{m=0}^{\infty} \Gamma(1+v m) \int_{-t}^{t}\left(\mathcal{H}_{1} \cos (v m \pi / 2)+i \mathcal{H}_{2} \operatorname{sign}(t-\tau) \sin (v m \pi / 2)\right) \\
& \times|t-\tau|^{-1-v m} \mathcal{G}(\tau, \mathcal{H}(\tau)) d \tau \mid \\
\leq & \frac{1}{2 \pi} \sum_{m=0}^{\infty} \Gamma(1+v m) \int_{-t}^{t}\left(\mathcal{H}_{1} \cos (v m \pi / 2)+i \mathcal{H}_{2} \operatorname{sign}(t-\tau) \sin (v m \pi / 2)\right) \\
& \times|t-\tau|^{-1-v m} G(\tau) d \tau \\
\leq & \frac{1}{2 \pi} \sum_{m=0}^{\infty} \Gamma(1+v m) K_{\lambda} G(t) .
\end{aligned}
$$

Thus,

$$
\mathcal{U}(t)-\mathcal{Q}(t)=\mathcal{U}(t)-\frac{1}{2 \pi} \sum_{m=0}^{\infty} \Gamma(1+v m)
$$




$$
\begin{aligned}
& \times \int_{-t}^{t}\left(\mathcal{H}_{1} \cos (\nu m \pi / 2)+i \mathcal{H}_{2} \operatorname{sign}(t-\tau) \sin (\nu m \pi / 2)\right) \\
& \times|t-\tau|^{-1-v m} \mathcal{G}(\tau, \mathcal{Q}(\tau)) d \tau
\end{aligned}
$$

By taking modulus on both sides, we have

$$
\begin{aligned}
\mid \mathcal{U}(t) & -\mathcal{Q}(t) \mid \\
\leq & \mid \mathcal{U}(t)-\frac{1}{2 \pi} \sum_{m=0}^{\infty} \Gamma(1+\nu m) \int_{-t}^{t}\left(\mathcal{H}_{1} \cos (v m \pi / 2)+i \mathcal{H}_{2} \operatorname{sign}(t-\tau) \sin (\nu m \pi / 2)\right) \\
& \times|t-\tau|^{-1-v m} \mathcal{G}(\tau, \mathcal{U}(\tau)) d \tau \mid \\
& +\mid \frac{1}{2 \pi} \sum_{m=0}^{\infty} \Gamma(1+\nu m) \int_{-t}^{t}\left(\mathcal{H}_{1} \cos (\nu m \pi / 2)-i \mathcal{H}_{2} \operatorname{sign}(t-\tau) \sin (\nu m \pi / 2)\right) \\
& \times|t-\tau|^{-1-v m} \mathcal{G}(\tau, \mathcal{U}(\tau))-\mathcal{G}(\tau, \mathcal{Q}(\tau)) d \tau \mid \\
\leq & \frac{1}{2 \pi} \sum_{m=0}^{\infty} \Gamma(1+\nu m) K_{\lambda} G(t)+\frac{L C}{2 \pi} \sum_{m=0}^{\infty} \Gamma(1+\nu m) \int_{-t}^{t}|t-\tau|^{-1-v m}|\mathcal{F}(\tau)-\mathcal{Q}(\tau)| d \tau
\end{aligned}
$$

By Grownwall's inequality, we obtain

$$
|\mathcal{U}(t)-\mathcal{Q}(t)| \leq W^{*} G(t)
$$

where $W^{*}=\left(\frac{K_{\lambda}}{2 \pi} \sum_{m=0}^{\infty} \Gamma(1+\nu m)\right) \exp \left(\frac{L C}{2 \pi} \sum_{m=0}^{\infty} \Gamma(1+\nu m) \int_{-t}^{t}|t-\tau|^{-1-\nu m} d \tau\right)$.

Therefore, equation (4.1) is stable in the sense of Hyers-Ulam-Rassias with respect to G on $(-t, t)$.

\section{Result}

Example 5.1 Consider the following:

$$
{ }^{\mathcal{A B R}} \mathcal{D}_{0}^{\frac{1}{2}} \mathcal{U}(t)=\frac{e^{-2 t}}{5}, \quad t \in(0,3)
$$

with $\nu=\frac{1}{2}, \beta=0, \mathcal{G}(t)=\frac{e^{-2 t}}{5}$.

Note that a function $\mathcal{U}_{1}(t)=e^{t}$ satisfies

$$
\left|\mathcal{A B R}^{\frac{1}{2}} e_{0}^{t}-\frac{e^{-2 t}}{5}\right| \leq \frac{1}{5}=\epsilon
$$

From equation (3.5), we obtain that the exact solution of equation (5.1) is

$$
\mathcal{U}_{\nu}(t)=\frac{1}{\pi} \sum_{m=0}^{\infty} \Gamma\left(1+\frac{1}{2} m\right) \int_{0}^{3}(t-\tau)^{-1-\frac{1}{2} m} \frac{e^{-2 \tau}}{5} d \tau
$$


By Theorem 3.1, problem (5.1) has a solution and it is stable in the Hyers-Ulam sense with

$$
\begin{aligned}
\left|\mathcal{U}_{1}(t)-\mathcal{U}_{v}(t)\right| & \leq \frac{1}{2 \pi} \sum_{m=0}^{\infty} \Gamma\left(1+\frac{1}{2} m\right)\left|\int_{0}^{3}(t-\tau)^{-1-\frac{1}{2} m} \mathcal{Y}_{1}(\tau) d \tau\right| \\
& \leq \frac{1}{10 \pi} \sum_{m=0}^{\infty} \Gamma\left(1+\frac{1}{2} m\right)\left|\int_{0}^{3}(t-\tau)^{-1-\frac{1}{2} m} d \tau\right| \\
& \leq \frac{1}{10 \pi} \sum_{m=0}^{\infty} \Gamma\left(1+\frac{1}{2} m\right)\left(\frac{3^{-\frac{m}{2}}}{\frac{m}{2}}\right), \\
\left|\mathcal{U}_{1}(t)-\mathcal{U}_{v}(t)\right| & \leq K \epsilon,
\end{aligned}
$$

where $K=\frac{1}{2 \pi} \sum_{m=0}^{\infty} \Gamma\left(1+\frac{1}{2} m\right)\left(\frac{3^{-\frac{m}{2}}}{\frac{m}{2}}\right)$. As such, our result can be applied to equation (5.1).

\section{Conclusion}

In this paper, the analysis of the results with respect to the fractional Fourier transform method is very efficient for solving linear and nonlinear FODE with Atangana and Baleanu fractional derivative in $\Phi(\mathbb{R})$. Different types of stability pertaining to such equations using fractional Fourier transform are established. We have effectively examined the existence and uniqueness solution of nonlinear FODE by using the Arzela-Ascoli theorem, Schaefer's fixed point theorem, as well as the Banach contraction principle. An illustrative example that demonstrates the applicability of the results has been included.

\section{Acknowledgements}

This research is made possible through financial support from the Phuket Rajabhat University, Thailand, and the Thailand Research Fund (RSA6280004). The authors are grateful to the Phuket Rajabhat University, Thailand, and the Thailand Research Fund (RSA6280004) for supporting this research.

Funding

The research is supported by the Phuket Rajabhat University, Thailand, and the Thailand Research Fund (RSA6280004).

\section{Availability of data and materials}

Data sharing is not applicable to this article as no datasets were generated or analysed during the current study.

\section{Competing interests}

The authors declare that they have no competing interests.

\section{Authors' contributions}

Funding acquisition, GR and PH; Conceptualization, GR and PH; Software, GR; Formal analysis, GR and PH; Methodology, GR and PH; Supervision, CPL, VG, AG and NG; Writing-original draft, GR and PH; Validation, GR, VG, AG and NG; Writingreview and editing, GR. All authors have read and agreed to the published version of the manuscript.

\section{Author details}

${ }^{1}$ Department of Mathematics, Phuket Rajabhat University, 83000 Phuket, Thailand. ${ }^{2}$ Department of Mathematics, Adhiyamaan College of Engineering, Hosur, Tamilnadu, India. ${ }^{3}$ Department of Mathematics, Government Arts and science College, Periyar University, Hosur, Tamilnadu, India. ${ }^{4}$ Department of Mathematics, Faculty of Science, Maejo University, Sansai, 50290 Chiang Mai, Thailand. ${ }^{5}$ Department of Mathematics, Sri Vidya Mandir Arts \& Science College, Katteri, Uthangarai, India. ${ }^{6}$ Department of Mathematical Sciences, Shibaura Institute of Technology, Omiya, 337-8570 Saitama, Japan. ${ }^{7}$ Institute for Intelligent Systems Research and Innovation, Deakin University, Waurn Ponds, 3216 VIC, Australia.

\section{Publisher's Note}

Springer Nature remains neutral with regard to jurisdictional claims in published maps and institutional affiliations. 


\section{References}

1. Rekhviashvili, S., Pskhu, A., Agarwal, P., Jain, S.: Application of the fractional oscillator model to describe damped vibrations. Turk. J. Phys. 43(3), 236-242 (2019)

2. Agarwal, P., Jain, S.: Further results on fractional calculus of Srivastava polynomials. Bull. Math. Anal. Appl. 3(2), 167-174 (2011)

3. Agarwal, P., Baltaeva, U., Alikulov, Y.: Solvability of the boundary-value problem for a linear loaded integro-differential equation in an infinite three-dimensional domain. Chaos Solitons Fractals 140, 110108 (2020)

4. Agarwal, P., Jain, S., Agarwal, S., Nagpal, M.: On a new class of integrals involving Bessel functions of the first kind. Commun. Numer. Anal. 2014, 1-7 (2014)

5. Al-Refai, M., Luchko, Y.: Maximum principle for the fractional diffusion equations with the Riemann-Liouville fractional derivative and its applications. Fract. Calc. Appl. Anal. 17(2), 483-498 (2014)

6. Bazhlekova, E., Bazhlekov, I.: Viscoelastic flows with fractional derivative models: computational approach by convolutional calculus of Dimovski. Fract. Calc. Appl. Anal. 17(4), 954-976 (2014)

7. Liu, Z., Li, X.: Approximate controllability of fractional evolution systems with Riemann-Liouville fractional derivatives. SIAM J. Control Optim. 53(4), 1920-1933 (2015)

8. Caputo, M., Fabrizio, M.: A new definition of fractional derivative without singular kernel. Prog. Fract. Differ. Appl. 1(2), 1-13 (2015)

9. Baleanu, D., Fernandez, A.: On some new properties of fractional derivatives with Mittag-Leffler kernel. Commun. Nonlinear Sci. Numer. Simul. 59, 444-462 (2018)

10. Shah, K., Abdeljawad, T., Mahariq, I., Jarad, F.: Qualitative analysis of a mathematical model in the time of COVID-19. BioMed Res. Int. 2020, 5098598 (2020)

11. Abdo, M.S., Shah, K., Wahash, H.A., Panchal, S.K.: On a comprehensive model of the novel coronavirus (COVID-19) under Mittag-Leffler derivative. Chaos Solitons Fractals 2020, 109867 (2020)

12. Alkahtani, B.S.T.: Chua's circuit model with Atangana-Baleanu derivative with fractional order. Chaos Solitons Fractals 89, 547-551 (2016)

13. Agarwal, P., Jain, S., Mansour, T.: Further extended Caputo fractional derivative operator and its applications. Russ. J. Math. Phys. 24(4), 415-425 (2017)

14. Weisstein, E.W.: Mittag-Leffler function. https://mathworld.wolfram.com/ (2003)

15. Agarwal, R.P.: A propos d'une note de M. Pierre Humbert. C. R. Acad. Sci. Paris 236(21), 2031-2032 (1953)

16. Sontakke, B.R., Kamble, G.P., Ul-Haque, M.M.: Some integral transform of generalized Mittag-Leffler functions. Int. J. Pure Appl. Math. 108(2), 327-339 (2016)

17. Agarwal, P., Chand, M., Baleanu, D., O'Regan, D., Jain, S.: On the solutions of certain fractional kinetic equations involving k-Mittag-Leffler function. Adv. Differ. Equ. 2018(1), 249 (2018)

18. Ulam, S.M.: A Collection of Mathematical Problems. New York, 29 (1960)

19. Hyers, D.H.: On the stability of the linear functional. Proc. Natl. Acad. Sci. USA 27(4), 222 (1941)

20. Rassias, T.M.: On the stability of the linear mapping in Banach spaces. Proc. Am. Math. Soc. 72(2), 297-300 (1978)

21. Obloza, M.: Hyers stability of the linear differential equation. Rocznik Nauk.-Dydakt. Prace Mat. 13, 259-270 (1993)

22. Alsina, C., Ger, R.: On some inequalities and stability results related to the exponential function. J. Inequal. Appl. 1998(4), 246904 (1998)

23. Rezaei, H., Jung, S.-M., Rassias, T.M.: Laplace transform and Hyers-Ulam stability of linear differential equations. J. Math. Anal. Appl. 403(1), 244-251 (2013)

24. Alqifiary, Q.H., Jung, S.-M.: Laplace transform and generalized Hyers-Ulam stability of linear differential equations. Electron. J. Differ. Equ. 2014, 80 (2014)

25. Ali, Z., Zada, A., Shah, K.: Ulam stability results for the solutions of nonlinear implicit fractional order differential equations. Hacet. J. Math. Stat. 48(4), 1092-1109 (2018)

26. Sher, M., Shah, K., Rassias, J.: On qualitative theory of fractional order delay evolution equation via the prior estimate method. Math. Methods Appl. Sci. 43(10), 6464-6475 (2020)

27. Ali, A., Samet, B., Shah, K., Khan, R.A.: Existence and stability of solution to a toppled systems of differential equations of non-integer order. Bound. Value Probl. 2017(1), 16 (2017)

28. Shah, K., Hussain, W.: Investigating a class of nonlinear fractional differential equations and its Hyers-Ulam stability by means of topological degree theory. Numer. Funct. Anal. Optim. 40(12), 1355-1372 (2019)

29. Shah, K., Shah, L., Ahmad, S., Rassias, J.M., Li, Y.: Monotone iterative techniques together with Hyers-Ulam-Rassias stability. Math. Methods Appl. Sci. (2019)

30. Agarwal, P., Ntouyas, S., Jain, S., Chand, M., Singh, G.: Fractional kinetic equations involving generalized k-Bessel function via Sumudu transform. Alex. Eng. J. 57(3), 1937-1942 (2018)

31. Unyong, B., Mohanapriya, A., Ganesh, A., Rajchakit, G., Govindan, V., Vadivel, R., Gunasekaran, N., Lim, C.P.: Fractional Fourier transform and stability of fractional differential equation on Lizorkin space. Adv. Differ. Equ. 2020(1), 578 (2020)

32. Mohanapriya, A., Ganesh, A., Gunasekaran, N.: The Fourier transform approach to Hyers-Ulam stability of differential equation of second order. J. Phys. Conf. Ser. 1597, 012027 (2020)

33. Mohanapriya, A., Ganesh, A., Rajchakit, G., Pinelas, S., Govindan, V., Unyong, B., Gunasekaran, N.: New generalization of Hermite-Hadamard type of inequalities for convex functions using Fourier integral transform. Thai J. Math. 18(3), 1051-1061 (2020)

34. Wiener, N.: Hermitian polynomials and Fourier analysis. J. Math. Phys. 8(1-4), 70-73 (1929)

35. Namias, V: The fractional order Fourier transform and its application to quantum mechanics. IMA J. Appl. Math. 25(3), 241-265 (1980)

36. McBride, A., Kerr, F.: On Namias's fractional Fourier transforms. IMA J. Appl. Math. 39(2), 159-175 (1987)

37. Liu, K., Wang, J., Zhou, Y., O'Regan, D.: Hyers-Ulam stability and existence of solutions for fractional differential equations with Mittag-Leffler kernel. Chaos Solitons Fractals 132, 109534 (2020)

38. Sene, N., Srivastava, G.: Generalized Mittag-Leffler input stability of the fractional differential equations. Symmetry 11(5), 608 (2019)

39. Lizorkin, P..: Generalized Liouville differentiation and the functional spaces $L_{p}^{r}\left(E_{n}\right)$. Imbedding theorems. Mat. Sb. 102(3), 325-353 (1963) 
40. Lizorkin, P...: Generalized Liouville differentiation and the method of multiplicators in imbedding theory for function classes. Math. Notes Acad. Sci. USSR 4(4), 771-779 (1968)

41. Samko, S.: Denseness of the spaces $\Phi(V)$ of Lizorkin type in the mixed $L_{p}\left(R_{n}\right)$-spaces. Stud. Math. 3(113), 199-210 (1995)

42. Zayed, A.I.: Fractional Fourier transform of generalized functions. Integral Transforms Spec. Funct. 7(3-4), 299-312 (1998)

43. Srivastava, H.M., Tomovski, Ž.: Fractional calculus with an integral operator containing a generalized Mittag-Leffler function in the kernel. Appl. Math. Comput. 211(1), 198-210 (2009)

44. Agarwal, R., Hristova, S., O'Regan, D.: Basic concepts of Riemann-Liouville fractional differential equations with non-instantaneous impulses. Symmetry 11(5), 614 (2019)

45. Abdeljawad, T., Baleanu, D.: Integration by parts and its applications of a new nonlocal fractional derivative with Mittag-Leffler nonsingular kernel. arXiv:1607.00262 (2016)

46. Fernandez, A., Baleanu, D.: On a new definition of fractional differintegrals with Mittag-Leffler kernel. arXiv:1807.1060 (2018)

47. Abdeljawad, T., Baleanu, D.: Integration by parts and its applications of a new nonlocal fractional derivative with Mittag-Leffler nonsingular kernel. arXiv:1607.00262 (2016)

48. Haleand, J., Lunel, S.: Introduction to Functional Differential Equations. Applied Mathematical Sciences. Springer, NewYork (1993)

49. Granas, A., Dugundji, J.: Fixed point theory. Bull. Am. Math. Soc. 41(2), 267-271 (2004)

50. Kilbas, A., Luchko, Y.F., Martinez, H., Trujillo, J.: Fractional Fourier transform in the framework of fractional calculus operators. Integral Transforms Spec. Funct. 21(10), 779-795 (2010)

51. Prudnikov, A., Brychkov, Y.A., Marichev, O.I.: Integrals and Series, Volume 1: Elementary Functions. Gordon \& Breach, New York (1986)

52. Yadav, D.K.: A new approach to ordering complex numbers. Int. J. Math. Sci. Eng. Appl. 2(3), 211-223 (2008)

53. Coen, C.S., Tassi, E.: A constructive and formal proof of Lebesgue's dominated convergence theorem in the interactive theorem prover Matita. J. Formaliz. Reason. 1(1), 51-89 (2008)

\section{Submit your manuscript to a SpringerOpen ${ }^{\circ}$ journal and benefit from:}

- Convenient online submission

- Rigorous peer review

- Open access: articles freely available online

- High visibility within the field

- Retaining the copyright to your article

Submit your next manuscript at $>$ springeropen.com 